\title{
Neutralization assay with SARS-CoV-1 and SARS-CoV-2 spike pseudotyped murine leukemia virions
}

Yue Zheng ${ }^{1}$, Erin T. Larragoite' ${ }^{1}$ Elizabeth S. C. P. Williams', Juan Lama², Isabel Cisneros², Julio C. Delgado ${ }^{1,3}$, Patricia Slev ${ }^{1,3}$, Jenna Rychert ${ }^{3}$, Emily A. Innis' ${ }^{1}$, Mayte Coiras ${ }^{4}$, Matthew T. Rondina ${ }^{5}$, Adam M. Spivak ${ }^{6}$ and Vicente Planelles ${ }^{1 *}$ (1)

\begin{abstract}
Background: Virus neutralization by antibodies is an important prognostic factor in many viral diseases. To easily and rapidly measure titers of neutralizing antibodies in serum or plasma, we developed pseudovirion particles composed of the spike glycoprotein of SARS-CoV-2 incorporated onto murine leukemia virus capsids and a modified minimal murine leukemia virus genome encoding firefly luciferase. This assay design is intended for use in laboratories with biocontainment level 2 and therefore circumvents the need for the biocontainment level 3 that would be required for replication-competent SARS-CoV-2 virus. To validate the pseudovirion assay, we set up comparisons with other available antibody tests including those from Abbott, Euroimmun and Siemens, using archived, known samples.

Results: 11 out of 12 SARS-CoV-2-infected patient serum samples showed neutralizing activity against SARS-CoV2-spike pseudotyped MLV viruses, with neutralizing titers-50 (NT $\left.{ }_{50}\right)$ that ranged from 1:25 to 1:1,417. Five historical samples from patients hospitalized for severe influenza infection in 2016 tested negative in the neutralization assay $\left(\mathrm{NT}_{50}<25\right)$. Three serum samples with high neutralizing activity against SARS-CoV-2/MLV pseudoviruses showed no detectable neutralizing activity $\left(\mathrm{NT}_{50}<25\right)$ against SARS-CoV-1/MLV pseudovirions. We also compared the semiquantitative Siemens SARS-CoV-2 lgG test, which measures binding of lgG to recombinantly expressed receptor binding domain of SARS-CoV-2 spike glycoprotein with the neutralization titers obtained in the pseudovirion assay and the results show high concordance between the two tests $\left(R^{2}=0.9344\right)$.
\end{abstract}

Conclusions: SARS-CoV-2 spike/MLV pseudovirions provide a practical means of assessing neutralizing activity of antibodies in serum or plasma from infected patients under laboratory conditions consistent with biocontainment level 2. This assay offers promise also in evaluating immunogenicity of spike glycoprotein-based candidate vaccines in the near future.

Keywords: COVID-19, Coronavirus, SARS, SARS-CoV-2, Neutralization assay, Pseudotyped virus, Spike, Murine leukemia virus, Antibody

*Correspondence: vicente.planelles@path.utah.edu

1 Department of Pathology, University of Utah School of Medicine, Salt Lake City, UT, USA

Full list of author information is available at the end of the article

\section{Introduction}

Coronaviruses are a group of enveloped RNA viruses with a positive-sense single-stranded RNA genome ranging from 26 to 32 kilobases, which can cause respiratory tract infections. In December 2019, a novel coronavirus known as severe acute respiratory syndrome coronavirus original author(s) and the source, provide a link to the Creative Commons licence, and indicate if changes were made. The images or other third party material in this article are included in the article's Creative Commons licence, unless indicated otherwise in a credit line to the material. If material is not included in the article's Creative Commons licence and your intended use is not permitted by statutory regulation or exceeds the permitted use, you will need to obtain permission directly from the copyright holder. To view a copy of this licence, visit http://creativecommons.org/licenses/by/4.0/. The Creative Commons Public Domain Dedication waiver (http://creativeco mmons.org/publicdomain/zero/1.0/) applies to the data made available in this article, unless otherwise stated in a credit line to the data. 
2 (SARS-CoV-2) was identified in China and has caused a global ongoing pandemic of coronavirus disease (COVID-19). To date, SARS-CoV-2 has spread to 188 countries (https://coronavirus.jhu.edu/). More than 45 million cases and 1,185,760 deaths have been reported globally at the time of this writing.

Enveloped viruses are known to efficiently package their core elements with heterologous envelope glycoproteins, giving rise to the so called 'pseudotypes' or 'pseudoviruses'. Many laboratories have successfully generated pseudotypes containing the core elements of HIV-1 [1] or MLV [2,3] and the envelope glycoproteins of vesicular stomatitis virus [4], murine leukemia virus [5], Lassa fever virus, ebola virus, coronavirus spike glycoproteins, and others (reviewed in [6]).

For a pseudotype virus, viral attachment [7], entry, and importantly, antibody binding and neutralization sensitivity are dependent on the membrane glycoprotein provided [6]. Using a defective MLV vector genome encoding firefly luciferase, and a packaging vector encoding MLV gag/pol, we describe the production of pseudovirus particles containing the spike glycoprotein of SARS-CoV-2. As controls, we also produced similar particles containing SARS-CoV-1, VSV-G or HIV-1 LAI gp160.

\section{Materials and methods \\ Cells}

HEK293FT cells, SupT1 cells and Huh7 cells were purchased from ATCC. HEK293FT and Huh7 cells were cultured in Dulbecco's modified Eagle's medium (DMEM) (Gibco, US) supplemented with 10\% FBS (Gibco, US) and $2 \mathrm{mM}$ L-glutamine (Gibco, US) at $37{ }^{\circ} \mathrm{C}$ with $5 \%$ $\mathrm{CO}_{2}$. SupT1 cells were cultured in RPMI supplemented with 10\% FBS and $2 \mathrm{mM}$ L-glutamine. HEK293T-ACE2 cells were cultured in DMEM with 10\% FBS, $2 \mathrm{mM}$ L-glutamine and $200 \mu \mathrm{g} / \mathrm{mL}$ hygromycin B (ThermoFisher, US). HEK293T-ACE2 cells were a gift from Adam Bailey and Emma Winkler (Washington University).

\section{Plasmids}

SV-Psi ${ }^{-}$-Env ${ }^{-}$-MLV [8], pHIV-1 LAI gp160 [9], pHCMVVSV-G [4] and pSIVmac gp130 [10] were previously described. L-LUC-SN was constructed by inserting the firefly luciferase gene within the polylinker of pLXSN (Clonetech, cat\# 631509). pSARS-CoV-1 was purchased from Sino Biologicals. pCAGGS expressing SARS-CoV-2 RBD was obtained from BEI Resources (cat\#NR-52309). The plasmid pcDNA3.1-SARS-2-S-C9 was a generous gift from Tom Gallagher and expresses a codonoptimized SARS-CoV-2 spike open reading frame with a deletion in the 19 carboxy-terminal amino acids (an endoplasmic reticulum retention signal) and addition of the C9 peptide TETSQVAPA, recognized by antibody $1 \mathrm{D} 4$.

\section{Production of pseudotyped MLV}

The plasmid SV-Psi ${ }^{-}-\mathrm{Env}^{-}$-MLV and L-LUC-SN were co-transfected with or without an envelope glycoprotein plasmid (pHCMV-VSV-G/pSARS-CoV-1/pSARS-CoV-2/ pHIV-1 LAI gp160) into HEK293FT cells using Lipofectamine $^{\mathrm{TM}} 3000$ (ThermoFisher, US). Cell supernatants containing viruses were collected after 2 days of transfection. Viruses were filtered through a $0.45 \mu \mathrm{m}$ filter (VWR, US) and centrifuged at $4{ }^{\circ} \mathrm{C}, 6500 \mathrm{rpm}$ for $18 \mathrm{~h}$ over a $20 \%$ sucrose cushion. Viruses were resuspended in $500 \mu \mathrm{L}$ cell culture medium and stored at $-80{ }^{\circ} \mathrm{C}$. We measured a $25 \%$ loss in infectivity due to one cycle of freeze/thaw for the SARS-CoV-2 pseudotype, and no loss for the VSV-G pseudotype.

\section{Pseudovirus infection}

HEK293FT, HEK293T-ACE2, and Huh7 cells were seeded in 96-well plates (ThermoFisher, US) the day before infection. SupT1 cells were added into a 96-well plate at the time of infection. $5 \times 10^{4}$ cells were added to each well. Pseudotyped MLV viruses were added to the pre-cultured cells. Cells were cultured at $37{ }^{\circ} \mathrm{C}$ with $5 \%$ $\mathrm{CO}_{2}$ for 2 days. All cells in each well were lysed and luciferase was measured using ONE-Glo ${ }^{\mathrm{TM}}$ Luciferase Assay reagent (Promega, US). RLUs are per well of a 96-well plate.

\section{Human samples}

Blood samples in this study were obtained from Associated Regional and University Pathologists, Inc. (ARUP) Laboratories. In all cases, samples were de-identified. Samples were tested for one or a combination of the following assays: Abbott Architect SARS-CoV-2 IgG, specific for nucleocapsid; Euroimmun Anti-SARS-CoV-2 ELISA (IgG), specific for the S1 domain of the spike glycoprotein; Siemens SARS-CoV-2 IgG, specific for RBD-binding IgG; PCR test specific for viral nucleic acid (ARUP Laboratories). Five pre-pandemic serum samples (Flu\#1-5) were archived and were from patients hospitalized for severe flu respiratory infections in 2016.

\section{Neutralization assay}

HEK293T-ACE2 cells were seeded in 96-well plates at $5 \times 10^{4}$ cells per well the day prior to infection. Sera were serially diluted in a volume of $50 \mu \mathrm{L}$ using DMEM with $10 \%$ FBS, $2 \mathrm{mM}$ L-glutamine and $200 \mu \mathrm{g} / \mathrm{mL}$ hygromycin $\mathrm{B}$ as diluent, and pre-incubated with $50 \mu \mathrm{L}$ of pseudotyped viruses at $37^{\circ} \mathrm{C}$ for $1 \mathrm{~h}$. For these infections, virus stocks were used at a dilution resulting in 50-200 RLU in the absence of serum. Cells were then infected with the 
serum/pseudovirion mixtures. Luciferase was measured $48 \mathrm{~h}$ post infection using ONE-Glo ${ }^{\mathrm{TM}}$ Luciferase Assay reagent. Neutralization titers $\mathrm{NT}_{50}, \mathrm{NT}_{80}$ and $\mathrm{NT}_{90}$ were calculated using Prism 8 (GraphPad, US).

\section{Results}

To generate pseudovirion particles, three plasmids were co-transfected into HEK293FT cells. The first plasmid was the packaging construct, SV-Psi ${ }^{-}-\mathrm{Env}^{-}-\mathrm{MLV}$; the second plasmid was L-LUC-SN, a minimal retroviral transfer vector encoding the firefly luciferase reporter gene; the third plasmid was an expression construct encoding one of the following membrane viral glycoproteins: SARS-CoV spike (hereafter referred to as SARS-CoV-1), SARS-CoV-2 spike, HIV-1 LAI gp160 and VSV-G. VSV-G pseudotyped virus is used as a positive control because of its high infectivity in most cell types. HIV-1 LAI gp160-pseudotyped virus is used as a negative control as it utilizes CD4 as a primary receptor, which is present in SupT1 cells but absent in HEK293FT.

Pseudotyped MLV viruses were tested on HEK293FT, HEK293T-ACE2, Huh7 and SupT1 cells. HEK293FT cells were used as a control cell line, which is known to lack susceptibility of coronavirus and HIV due to the absence of both ACE2 and CD4. As expected, VSV-G pseudotyped viruses infected all cell types and showed the highest infectivity (Fig. 1). HIV-1 LAI gp160-pseudotyped viruses only infected SupT1 cells. Both SARS-CoV-1 spike-pseudotyped and SARS-CoV-2-spike pseudotyped viruses infected HEK293T-ACE2 and Huh7 cells.

Since HEK293T-ACE2 cells showed the highest susceptibility to both SARS-CoV-1 and SARS-CoV-2 pseudotyped MLV viruses, further experiments were all performed in HEK293T-ACE2 cells. The ultimate goal of our studies was to develop a virus neutralization test based on the above pseudotyped virus. To test for neutralization activity, serum samples from 12 de-identified COVID-19 patients were tested for their ability to neutralize pseudotyped MLV viruses. Samples 1-6 were sera from patients who were tested for nucleocapsid-specific IgG (Abbott; samples 1, 8, 9 and 12), spike-specific IgG (Euroimmun; samples 2, 4, 5, 6, 8 and 9) or Nucleic Acid Amplification test (ARUP Laboratories; samples 3, and 8-11).

As a positive control and also as a standard to monitor variability between neutralization experiments, we used recombinant soluble receptor binding domain (RBD) from SARS-CoV-2 spike protein. We produced this protein via transient transfection in HEK293FT cells using a mammalian expression vector (pCAGGS) encoding amino acids 319 to 542 of from SARS-CoV-2 S1, encompassing the RBD. The apparent $\mathrm{NT}_{50}$ of $\mathrm{RBD}$ against SARS-CoV-2/MLV pseudotype was 1:244 (Fig. 2). As a negative control for neutralization, the surface glycoprotein from the simian immunodeficiency virus, SIVmac

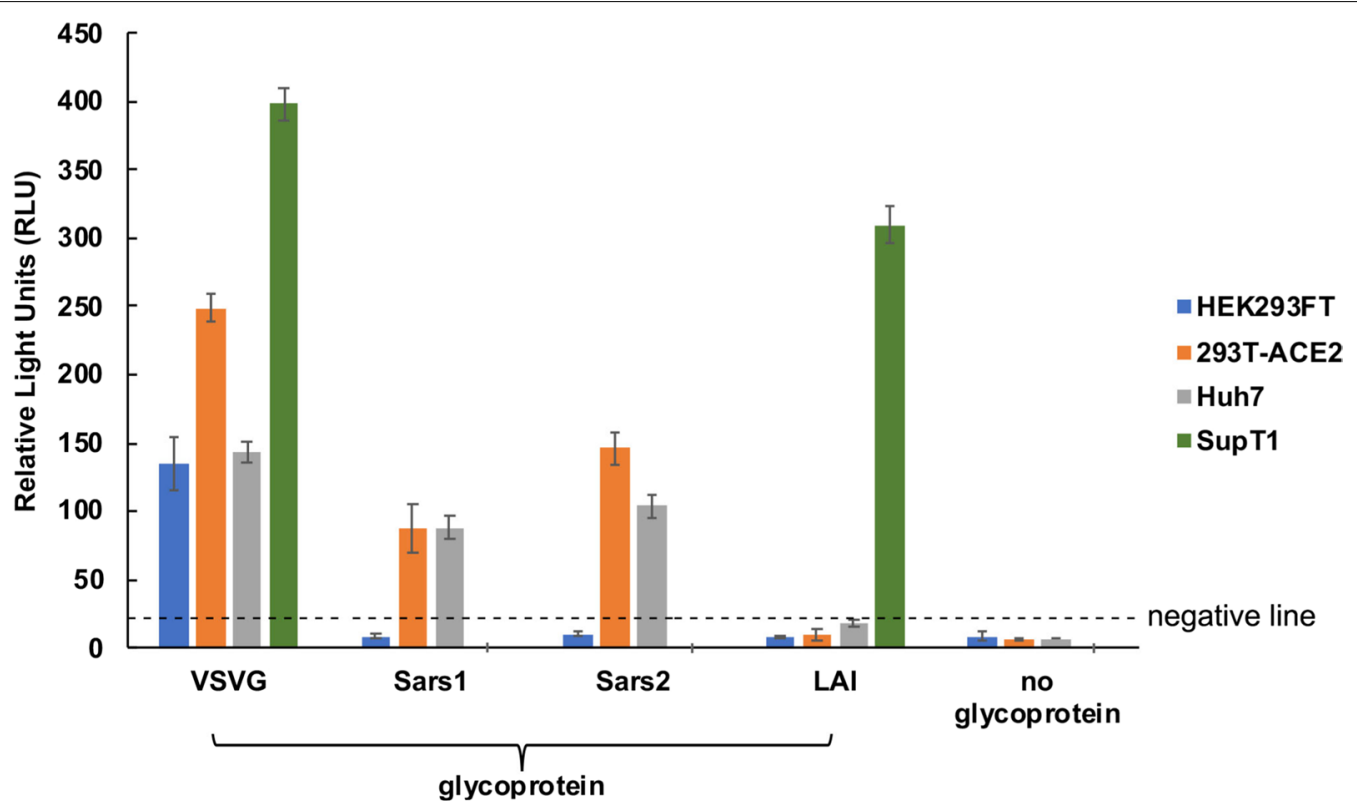

Fig. 1 Infectivity of pseudotyped MLV Viruses. SARS-CoV-2 spike pseudotyped MLV viruses as well as VSV-G, SARS-CoV-1 spike, and HIV-1 LAlgp160 pseudotyped MLV viruses were tested on HEK293FT, HEK293T-ACE2, Huh7 and SupT1 cells. $100 \mu \mathrm{L}$ of undiluted virus (except for VSV-G pseudotype, which was diluted 1:100) was mixed with $100 \mu \mathrm{L}$ of medium and added to cells. Luciferase was measured at 2 days post-infection and values are per well of a 96-well plate. Negative line indicates mean +3SD of luciferase values obtained with virions devoid of glycoprotein 


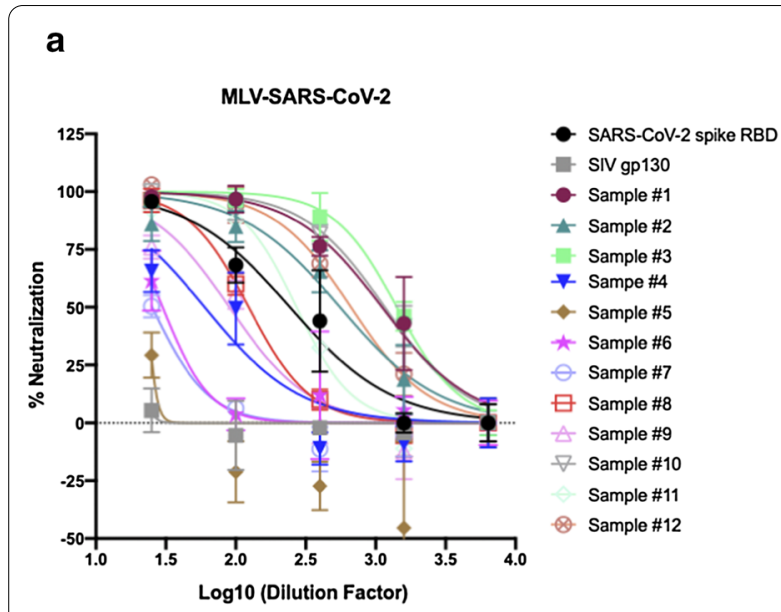

\begin{tabular}{|c|c|c|c|c|c|c|}
\hline \multicolumn{4}{|c|}{ MLV-Sars-CoV-2 } & \multirow[b]{3}{*}{ Abbott } & \multirow[b]{3}{*}{ Euroimmun } & \multirow[b]{3}{*}{ PCR } \\
\hline & & Im dilut & & & & \\
\hline & NT50 & NT80 & NT90 & & & \\
\hline SARS-CoV-2 RBD & 244 & 76 & 38 & & & \\
\hline SIV gp130 & $<25$ & $<25$ & $<25$ & & & \\
\hline Sample \#1 & 1110 & 405 & 224 & 5.6 &.- & - \\
\hline Sample \#2 & 557 & 180 & 92 & -- & 9.7 & - \\
\hline Sample \#3 & 1417 & 691 & 454 & -- & -- & + \\
\hline Sample \#4 & 60 & $<25$ & $<25$ & -- & 8.8 & - \\
\hline Sample \#5 & $<25$ & $<25$ & $<25$ & -- & 2.8 & - \\
\hline Sample \#6 & 30 & $<25$ & $<25$ & -- & 6.6 & - \\
\hline Sample \#7 & 25 & $<25$ & $<25$ & -- & -- & - \\
\hline Sample \#8 & 124 & 61 & 41 & 4.5 & 4.4 & + \\
\hline Sample \#9 & 90 & 36 & $<25$ & $<1.4$ & 1.1 & + \\
\hline Sample \#10 & 1162 & 460 & 268 & -- & -- & + \\
\hline Sample \#11 & 272 & 144 & 99 & -- & -- & + \\
\hline Sample \#12 & 667 & 276 & 165 & $<1.4$ & -- & - \\
\hline
\end{tabular}

Fig. 2 Neutralizing activity of COVID-19 patient sera against and SARS-CoV-2 pseudotyped MLV. a Serum samples from COVID-19 patients were pre-incubated with SARS-CoV-2 spike pseudotyped MLV at $37^{\circ} \mathrm{C}$ for $1 \mathrm{~h}$. Sera and virus mixture were then incubated with HEK293T-ACE2 cells for 2 days. SARS-CoV-2 spike RBD was used as a positive control. SIV gp130 was used as a negative control. Luciferase was measured to assess infection. b Neutralization titer 50,80 and $90\left(\mathrm{NT}_{50}, \mathrm{NT}_{80}, \mathrm{NT}_{90}\right)$ were calculated as the reciprocal of the dilution resulting in 50,80 and $90 \%$ neutralization, respectively. ELISA tests by Abbott and Euroimmun are not quantitative. Positive samples for the Abbott test are $\geq 1.4$ and for the Euroimmun test $\geq 1.1$

gp130 [10], was similarly produced by mammalian cell transfection.

As shown in Fig. 2, 11 out of 12 patient serum samples showed neutralizing activity against SARS-CoV2-spike pseudotyped MLV viruses, with neutralizing titers-50 $\left(\mathrm{NT}_{50}\right)$ that ranged from 1:25 to 1:1,417. Eight out of the 12 samples displayed detectable $\mathrm{NT}_{80}$ and 7 samples displayed detectable $\mathrm{NT}_{90}$. We also tested five historical samples from patients who were hospitalized for severe influenza infection in 2016. We tested the historical samples at the 1:25 dilution only and reported \% neutralization. All of these samples tested negative in our neutralization assay (Fig. 3).

To test for specificity of neutralization, we asked whether neutralizing antibodies from SARS-CoV-2 patients would exhibit cross-reactivity against a pseudotype expressing SARS-CoV-1 (Fig. 4). We tested samples $\# 1,2$ and 3, which had high neutralization titers. None of these sera had detectable neutralizing activity $\left(\mathrm{NT}_{50}<25\right)$ against the SARS-CoV-1 pseudotype, which is consistent with previous reports [11-13].

It has been proposed that most neutralizing antibodies against SARS-CoV-2 act by binding the RBD of the spike glycoprotein and blocking binding to ACE-2. The Siemens SARS-CoV-2 IgG assay measures binding of IgG to recombinant RBD [14]. We wished to assess the potential correlation between our pseudovirion neutralization test and Siemens SARS-CoV-2 IgG. For this purpose, we used 6 samples and tested them in parallel in both assays. We then plotted the $\mathrm{NT}_{50}$ titer from the pseudovirion test against the Anti-RBD IgG index provided by the Siemens test (Fig. 5). As shown on Fig. 5, both assays can be fitted to a linear regression model with an $R^{2}=0.93$.

\section{Discussion}

The importance of neutralizing antibodies in many viral infections cannot be overstated. The presence of neutralizing antibodies due to previous infection or vaccination is in many cases expected to protect the individual from future infection and/or from severe illness. While this has been documented for many other pathogens, how neutralizing antibodies will impact SARS-CoV-2 infection and disease severity is still unclear. The availability of tests that measure antibody neutralization in the setting of SARS-CoV-2 will lead to a better understanding of the mechanisms of pathogenesis and how the immune system reacts to the presence of the virus in the organism.

Ideally, vaccines against SARS-CoV-2 will become available toward the end of 2020. For vaccines that are based on the spike glycoprotein, studies measuring antibody neutralization, together with evaluation of cellular immunity, will help to better understand and predict vaccine effectiveness. Specifically, it is imperative that we understand whether neutralizing antibodies can be correlates of protection against progression to severe disease; and, if so, whether we can establish a threshold of neutralization activity above which protection is likely. Additionally, we should be able to establish whether vaccine-induced immunity is comparable to natural immunity from infection in terms of protection from infection and disease severity. 


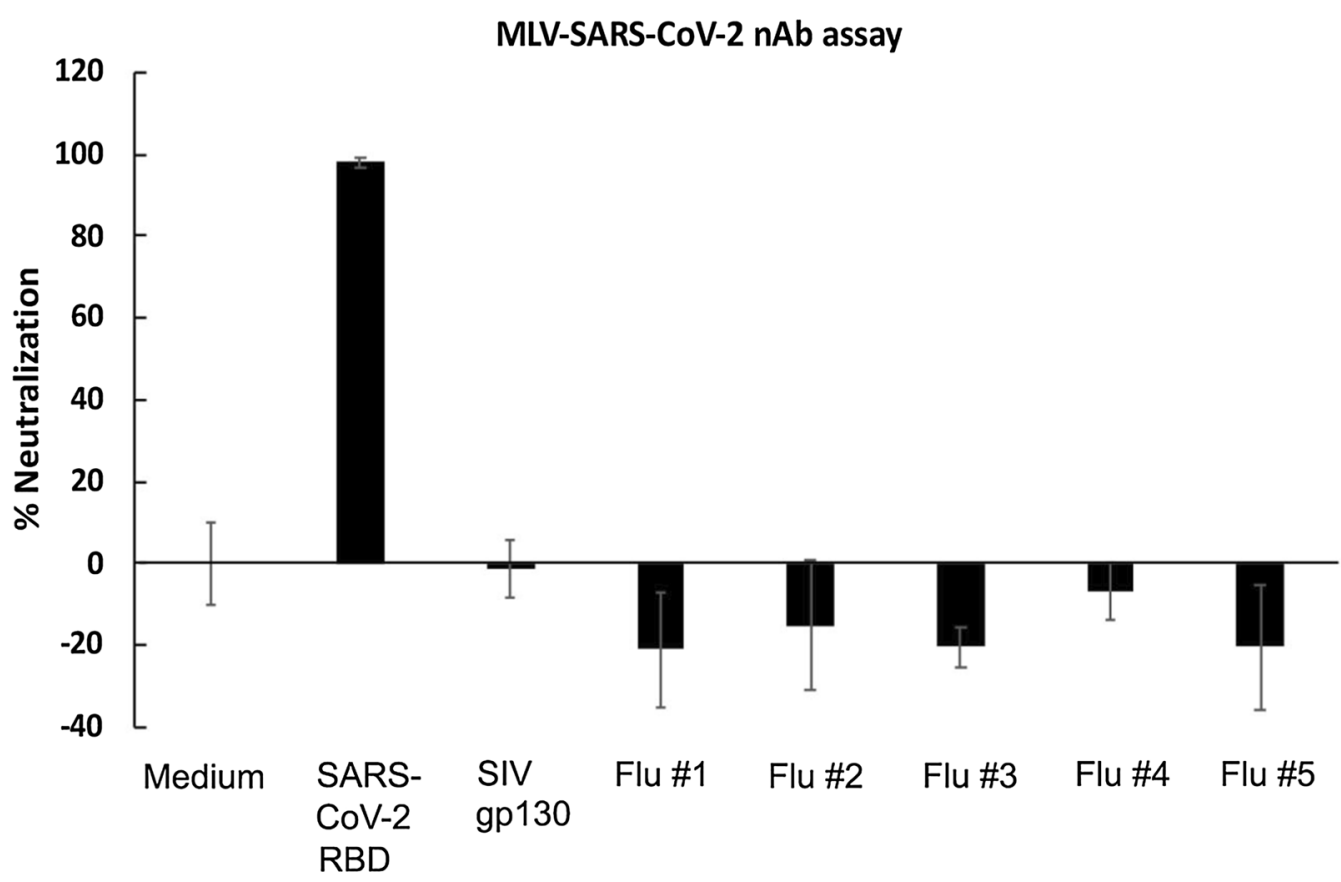

Fig. 3 Sera from hospitalized flu patients had no neutralizing activity against SARS-CoV-2 pseudovirions. Cryopreserved serum samples from hospitalized flu patients from 2016 were pre-incubated with SARS-CoV-2 spike pseudotyped MLV at $37^{\circ} \mathrm{C}$ for $1 \mathrm{~h}$. Serum and virus mixture were then incubated with HEK293T-ACE2 cells for 2 days. SARS-CoV-2 RBD was used as a positive control. SIV gp 130 was used as a negative control

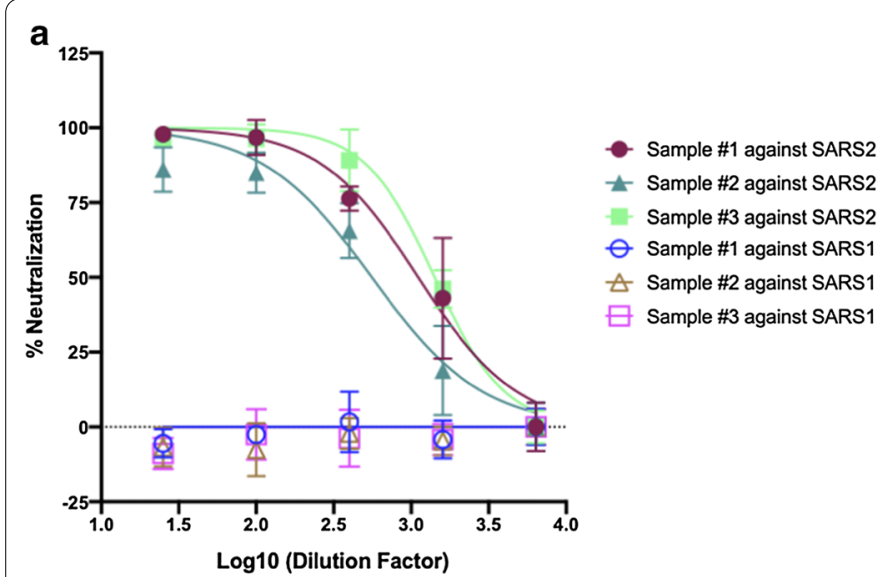

\section{b}

\begin{tabular}{|c|c|c|c|c|}
\hline & \multicolumn{4}{|c|}{ Serum dilution } \\
\hline & \multicolumn{2}{|c|}{ MLV-SARS-CoV2 } & \multicolumn{2}{c|}{ MLV-SARS-CoV1 } \\
\hline & NT50 & NT80 & NT50 & NT80 \\
\hline Sample \#1 & 1110 & 405 & $<25$ & $<25$ \\
\hline Sample \#2 & 557 & 180 & $<25$ & $<25$ \\
\hline Sample \#3 & 1417 & 691 & $<25$ & $<25$ \\
\hline
\end{tabular}

Fig. 4 Neutralizing activity of COVID-19 patient serum against SARS-CoV-1 pseudovirions. a Serum from COVID-19 patients were pre-incubated with SARS-CoV-1 spike pseudotyped MLV at $37^{\circ} \mathrm{C}$ for $1 \mathrm{~h}$. Serum and virus mixture were then incubated with HEK293T-ACE2 cells. b NT 50 and NT 80 were calculated as above. Samples \#1, 2 and 3 were tested previously (Fig. 2)

Antibody neutralization can also be a critical factor when deciding on potential sources of convalescent immune plasma as this approach is able to provide immediate immunity in susceptible individuals [15]. In one study, a single dose of $200 \mathrm{~mL}$ of convalescent plasma with an antibody titer of 1:640 was transfused to 10 patients along with antiviral agents and supportive measures [16]. Symptoms in all of patients, especially fever, cough, shortness of breath, and chest pain, disappeared or largely improved one to three days after plasma infusion [16]. Being able to evaluate the titers of neutralizing antibodies in convalescent plasma sources will allow for selection of samples with highest likelihood of success. 


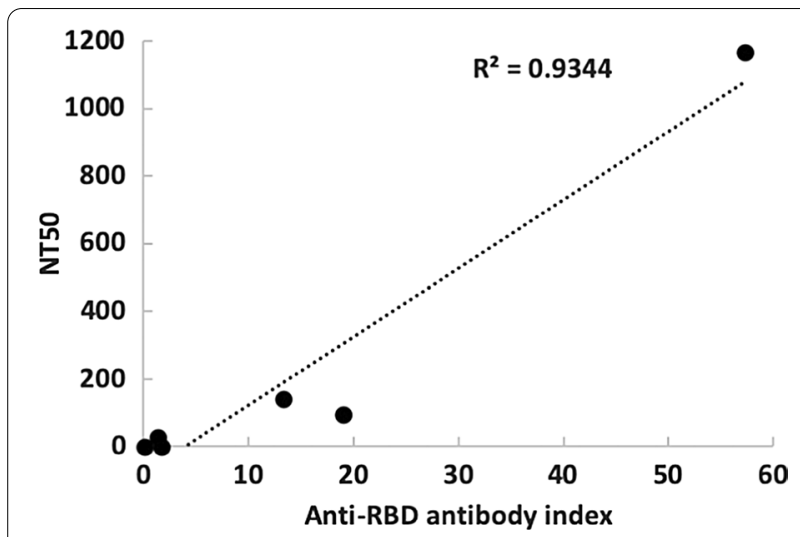

Fig. 5 Comparison between MLV pseudotype neutralization and the Siemens SARS-CoV-2 lgG assay. Siemens SARS-CoV-2 IgG assay measuring $\mathrm{IgG}$ binding to recombinant receptor binding domain (horizontal axis) was compared by linear regression to the MLV pseudotype assay (vertical axis). Samples used in this experiment were \#8 (shown in Fig. 2) and \#13 and \#15-18, not used in previous figures

\section{Conclusions}

In summary, we have developed a simple and rapid assay based on pseudovirion particles, which allows for specific measurement of neutralizing titers in plasma against SARS-CoV-2 in the context of biocontainment level 2 laboratories.

\section{Abbreviations}

SARS: Severe acute respiratory syndrome; SARS-CoV-2: Severe acute respiratory syndrome coronavirus 2; COVID-19: Coronavirus disease 2019; DMEM: Dulbecco's modified Eagle's medium; FBS: Fetal bovine serum; RBD: Receptor binding domain; NT: Neutralizing titer; MLV: Murine leukemia virus; VSV-G: Vesicular stomatitis virus glycoprotein; HIV: Human immunodeficiency virus; ACE2: Angiotensin Converting Enzyme 2.

\section{Acknowledgements}

The construct pcDNA3.1-SARS-2-S-C9 was a generous gift from Tom Gallagher at Loyola University. Human Codon Optimized HEK293T-ACE2 cells were kindly provided by Adam Bailey and Emma Winkler in the laboratory of Michael Diamond at Washington University in St. Louis School of Medicine. We also wish to thank Eloisa Yuste for helpful technical suggestions.

\section{Authors' contributions}

YZ, ETL, EAI, ESCPW conducted experiments. JL, IC, MC, AMS and VP designed the study. JCD, PS, JR and MTR selected and contributed archived samples. All authors read and approved the final manuscript.

\section{Funding}

This research was supported by a seed grant from the University of Utah Vice President for Research and the Immunology, Inflammation, and Infectious Disease Initiative. V.P. and M.C. were supported by NIH grant Al143567-01.

\section{Availability of data and materials}

Plasmid constructs and methodology are available upon request. Aliquots of plasma and serum are in limiting quantities and may be available depending on amount requested.

\section{Ethics approval and consent to participate}

We used de-identified, archived serum samples throughout the study.

\section{Consent for publication}

All authors have agreed to publication of the manuscript.

\section{Competing interests}

The authors declare no competing interests.

\section{Author details}

${ }^{1}$ Department of Pathology, University of Utah School of Medicine, Salt Lake City, UT, USA. ${ }^{2}$ RetroVirox, Inc., San Diego, CA, USA. ${ }^{3}$ Associated Regional and University Pathologists (ARUP) Laboratories, Salt Lake City, UT, USA. ${ }^{4}$ AIDS Immunopathology Unit, National Center of Microbiology (CNM), Instituto de Salud Carlos III, Madrid, Spain. ${ }^{5}$ Department of Human Genetics, University of Utah School of Medicine, Salt Lake City, UT, USA. ${ }^{6}$ Department of Medicine, University of Utah School of Medicine, Salt Lake City, UT, USA.

Received: 15 September 2020 Accepted: 16 December 2020

Published online: 04 January 2021

\section{References}

1. Ou X, Liu Y, Lei X, Li P, Mi D, Ren L, Guo L, Guo R, Chen T, Hu J, et al. Characterization of spike glycoprotein of SARS-CoV-2 on virus entry and its immune cross-reactivity with SARS-CoV. Nat Commun. 2020;11:1620.

2. Walls AC, Park YJ, Tortorici MA, Wall A, McGuire AT, Veesler D. Structure, function, and antigenicity of the SARS-CoV-2 spike glycoprotein. Cell. 2020;181:281-92 e286.

3. Zhang L, Jackson CB, Mou H, Ojha A, Rangarajan ES, Izard T, Farzan M, Choe $\mathrm{H}$. The D614G mutation in the SARS-CoV-2 spike protein reduces S1 shedding and increases infectivity. bioRxiv. 2020.

4. Akkina RK, Walton RM, Chen ML, Li QX, Planelles V, Chen IS. High-efficiency gene transfer into CD34+ cells with a human immunodeficiency virus type 1-based retroviral vector pseudotyped with vesicular stomatitis virus envelope glycoprotein G. J Virol. 1996;70:2581-5.

5. Planelles V, Bachelerie F, Jowett JB, Haislip A, Xie Y, Banooni P, Masuda T, Chen IS. Fate of the human immunodeficiency virus type 1 provirus in infected cells: a role for vpr. J Virol. 1995;69:5883-9.

6. Steffen I, Simmons G. Pseudotyping viral vectors with emerging virus envelope proteins. Curr Gene Ther. 2016;16:47-55.

7. Bosch BJ, van der Zee R, de Haan CA, Rottier PJ. The coronavirus spike protein is a class I virus fusion protein: structural and functional characterization of the fusion core complex. J Virol. 2003;77:8801-11.

8. Landau NR, Littman DR. Packaging system for rapid production of murine leukemia virus vectors with variable tropism. J Virol. 1992;66:5110-3.

9. Bosque A, Planelles V. Induction of HIV-1 latency and reactivation in primary memory CD4+ T cells. Blood. 2009;113:58-65.

10. Planelles V, Haigwood NL, Marthas ML, Mann KA, Scandella C, Lidster WD, Shuster JR, Van Kuyk R, Marx PA, Gardner MB, et al. Functional and immunological characterization of SIV envelope glycoprotein produced in genetically engineered mammalian cells. AIDS Res Hum Retrovir. 1991;7:889-98.

11. Yi C, Sun X, Ye J, Ding L, Liu M, Yang Z, Lu X, Zhang Y, Ma L, Gu W, et al. Key residues of the receptor binding motif in the spike protein of SARS-CoV-2 that interact with ACE2 and neutralizing antibodies. Cell Mol Immunol. 2020:17:621-30.

12. Lv H, Wu NC, Tsang OT, Yuan M, Perera R, Leung WS, So RTY, Chan JMC, Yip GK, ChikTSH, et al. Cross-reactive antibody response between SARSCoV-2 and SARS-CoV infections. bioRxiv. 2020.

13. Ju B, Zhang Q, Ge J, Wang R, Sun J, Ge X, Yu J, Shan S, Zhou B, Song S, et al. Human neutralizing antibodies elicited by SARS-CoV-2 infection. Nature. 2020:584:115-9.

14. Trabaud MA, Icard V, Milon MP, Bal A, Lina B, Escuret V. Comparison of eight commercial, high-throughput, automated or ELISA assays detecting SARS-CoV-2 IgG or total antibody. J Clin Virol. 2020;132:104613.

15. Kotta S, Aldawsari HM, Badr-Eldin SM, Alhakamy NA, Md S, Nair AB, Deb PK. Combating the Pandemic COVID-19: Clinical Trials, Therapies and Perspectives. Front Mol Biosci. 2020;7:606393.

16. Duan K, Liu B, Li C, Zhang H, Yu T, Qu J, Zhou M, Chen L, Meng S, Hu Y, et al. Effectiveness of convalescent plasma therapy in severe COVID-19 patients. Proc Natl Acad Sci USA. 2020;117:9490-6.

\section{Publisher's Note}

Springer Nature remains neutral with regard to jurisdictional claims in published maps and institutional affiliations. 